\title{
SMARCAL1 Mutations: A Cause of Prepubertal Idiopathic Steroid-resistant Nephrotic Syndrome
}

\author{
MIROSLAV ŽIVIČNJAK, DORIS FRANKE, MARTIN ZENKER, JULIANE HOYER, THOMAS LÜCKE, LARS PAPE, \\ AND JOCHEN H.H. EHRICH
}

\author{
Department of Pediatric Nephrology [M.Z., D.F., T.L., L.P., J.H.H.E.], Hannover Medical School, D-30623 Hannover, Germany; Institute \\ of Human Genetics [M.Z., J.H.], Friedrich-Alexander University Erlangen-Nuremberg, D-91054 Erlangen, Germany
}

\begin{abstract}
Schimke immuno-osseous dysplasia (SIOD) is a rare autosomal-recessive multisystem disorder with disproportionate growth failure, impaired $\mathrm{T}$ cell function, and steroid-resistant nephrotic syndrome. Recently, we presented the typical anthropometric features of SIOD. We now present data on two siblings who were initially classified as suffering from familial steroid-resistant nephrotic syndrome of unknown genetic origin. Apart from growth failure, no syndrome-specific symptoms were found until the age of $10 \mathrm{y}$. However, serial anthropometric examinations showed the development of a SIOD-like pattern with a decreased ratio of trunk to leg length in early adolescence. The growth pattern was significantly different from that seen in children with chronic renal failure of other origins. In prepuberty the siblings had proportionate short stature but developed disproportion only during adolescence. Molecular genetic analysis revealed compound heterozygosity for a known and a new mutation in the SMARCAL1 gene. Conclusion: the disease spectrum associated with SMARCAL1 mutations includes previously undescribed milder phenotypes that may be clinically overlooked, particularly before puberty. Serial anthropometric assessment can eventually identify patients with a growth pattern similar to that of SIOD. These patients should be tested for SMARCAL1 mutations to avoid overtreatment with immunosuppressive agents. (Pediatr Res 65: 564-568, 2009)
\end{abstract}

Key Words: Schimke immuno-osseous dysplasia (SIOD), anthropometry, growth pattern, chronic kidney disease, steroid-resistant nephrotic syndrome

$S^{c}$ chimke immuno-osseous dysplasia (SIOD, MIM 242900) is an autosomal-recessive multisystem disorder with prominent disproportionate growth failure, progressive steroid-resistant nephrotic syndrome with focal and segmental glomerulosclerosis, immunodeficiency, and life-limiting cerebral vascular complications (1-5). It is caused by mutations of SMARCAL1, a gene encoding a putative chromatin remodelling protein of unknown function (4). Patients with severe SIOD develop nephrotic syndrome and renal insufficiency by preschool age and suffer from life-threatening neurologic complications such as transient ischemic attacks or cerebral infarctions. In contrast, those with milder disease do not develop cerebral vascular complications until at least early adulthood (1-4,6-7).

The key clinical findings in SIOD are progressive renal failure and disproportionate growth failure (2). Patients with

Received August 8, 2008; accepted November 26, 2008

Corresponding author: Miroslav Živičnjak, Ph.D., Department of Pediatrics, Hannover Medical School, Carl-Neuberg Str. 1, D-30623 Hannover, Germany, Phone: +49-511532 6599, Fax: +49-511-532 3911; e-mail: zivicnjak.miroslav@mh-hannover.de

Financial Support: No financial assistance was received in support of this study.
SIOD are not only shorter than children with chronic kidney disease (CKD), but also have a distinctive growth pattern of better preserved leg than trunk length (5).

Among 197 female patients with CKD undergoing routine annual anthropometric measurement, we identified two siblings with steroid-resistant nephrotic syndrome who had a growth pattern atypical of nonsyndromic CKD patients but typical of SIOD. Molecular testing confirmed the presence of biallelic SMARCAL1 mutations.

\section{PATIENTS AND METHODS}

Patients. The two female siblings were identified by routine anthropometric measurements to have a growth pattern atypical of nonsyndromic CKD patients. The growth data of the two index patients was compared with anthropometric data of 197 female patients (658 annual measurements in the age range: 2-21 y) with CKD stage 2 and higher (8) undergoing annual investigations from May 1998 to September 2007. The study was approved by the Ethics Committee of Hannover Medical School and written informed consent was obtained from the parents. The different underlying primary kidney diseases of these 197 children are listed in Table 1.

Anthropometry. Four parameters of longitudinal growth were determined as recommended by the International Biologic Program (9): body height stature (HT), sitting height (SH), total arm length (AL), and leg length (LL). Measurements were performed using standardized equipment (Dr Keller I Stadiometer, Limbach-Oberfrohna, Germany; Siber Hegner Anthropometer, Zürich, Switzerland). The accuracy of the measurements was within $1 \mathrm{~mm}$.

Molecular genetics. DNA of both patients was extracted from venous blood samples according to standard techniques. The entire coding sequence, including exon-intron boundaries of the SMARCAL1 gene, was analyzed by bidirectional direct sequencing on an automated capillary sequencer (ABI3730, Applied Biosystems) according to the manufacturer's instructions. Primer sequences and PCR conditions are available on request.

Statistical analyses. $Z$ scores (SDS) were performed based on reference limits derived from 2,648 healthy females aged 3 to $18 \mathrm{y}$ (10). SDS values for $\mathrm{HT}, \mathrm{SH}, \mathrm{AL}$ and LL were calculated according to the equation: $S D S=\left(x_{i}-\right.$ $\left.x_{s}\right) / S D$, where $x_{i}$ is the individual value of patient, and $x_{s}$ and $S D$ are the mean and SD values for age-matched healthy girls. The normality of distribution of SDS values for HT, SH, AL and LL for CKD female patients, was evaluated by the Kolmogornov-Smirnov test for each age cohort. As the distribution of each anthropometric parameter did not differ significantly from normal, we applied parametric methods for our analysis.

\section{RESULTS}

Clinical findings. The two index patients are sisters. The mother and father as well as the oldest daughter of the family

Abbreviations: AL, arm length; CKD, chronic kidney disease; CSA, cyclosporine A; FSGS, focal segmental glomerulosclerosis; HT, body height stature; LL, leg length; MCNS, minimal glomerular lesions; SH, sitting height; SIOD, Schimke immuno-osseous dysplasia; SRNS, steroid-resistant nephrotic syndrome 
Table 1. The underlying primary diseases of 197 girls with chronic kidney disease stages 2-5

\begin{tabular}{lcc}
\hline \multicolumn{1}{c}{ Diagnosis } & $\begin{array}{c}\text { No. of } \\
\text { patients }\end{array}$ & $\begin{array}{c}\text { \% of all } \\
\text { patients }\end{array}$ \\
\hline Glomerulonephritis & 46 & 23.5 \\
Renal Hypoplasia/Dysplasia & 38 & 19.3 \\
Nephronophthisis & 28 & 14.2 \\
HUS & 19 & 9.6 \\
Obstruktive Uropathy/ & 17 & 8.6 \\
Tubulointerstitial disease & & \\
ARPKD & 16 & 8.1 \\
Cystinosis/Hyperoxaluria & 16 & 8.1 \\
Other & 17 & 8.6 \\
Total & 197 & 100 \\
\hline
\end{tabular}

are healthy. At the ages of 4 and $6 y$, respectively, the two siblings developed a steroid-resistant nephrotic syndrome. Renal biopsy was performed in the elder of the two girls and revealed absence of foot processes and minimal changes in 18 glomeruli. Neither steroid therapy, nor cyclosporine A (CSA) or ACE inhibitor therapy were able to induce remission. Apart from growth failure, no syndrome-specific symptoms such as hair, skin, and facial characteristics, bone abnormalities or laboratory determined immune defects were found until the age of $10 \mathrm{y}$.

Following the development of terminal renal failure at the age of $10 \mathrm{y}$, the elder sibling (patient 1 ) was treated with peritoneal dialysis until she received a maternal kidney transplant a year later. Transplant function has remained stable with a serum creatinine of $112 \mu \mathrm{M}$ while receiving CSA, mycophenolate mofetil and prednisolone. Proteinuria did not recur. After initiation of immunosuppressive therapy, she developed severe, therapy-resistant papillomas on her face, feet and hands leading to psychosocial problems and reactive depression. Due to the therapy-resistant papillomas, immunosuppression was reduced to CSA monotherapy. Because of a high EBV viral load, she was switched to sirolimus at the age of $14 \mathrm{y}$. At the age of $15 \mathrm{y}$, she developed ocular varicella zoster and herpes encephalitis leading to a left-sided Horner syndrome. At $19 \mathrm{y}$, she was hospitalized for treatment of sepsis. She also suffered from progressive hip dysplasia leading to severe pain requiring continuous treatment with buprenorphine. She did not develop signs or symptoms of cerebral ischemia.
When the younger sibling (patient 2) developed steroidresistant nephrotic syndrome, neither renal biopsy nor extensive immunosuppressive therapy were initiated because of the similarity of her disease to that of her sister. Beginning at $8 \mathrm{y}$, her renal failure was treated with peritoneal dialysis until she received a cadaveric renal transplant a year later. Her initial immunosuppressive therapy, basiliximab, CSA, mycophenolate mofetil and prednisolone, maintained stable transplant function with a serum creatinine of $65 \mu \mathrm{M}$ without recurrence of proteinuria. However, her course was complicated by CMV, varicella zoster, helicobacter, and enterococci infections. Due to cyclosporine nephrotoxicity, her immunosuppression was switched to everolimus and low-dose CSA. Shortly after she developed enterobacteriacea sepsis complicated by cardiac arrest. Her immunosuppression was then changed to CSA monotherapy with C2-levels of 350-500 $\mathrm{ng} / \mathrm{mL}$. Subsequently she was noted to have cyclic lymphopenia. She also developed papillomas on her hands, although not as severe as her older sister. She was diagnosed with bilateral hip dysplasia at the age of $13 \mathrm{y}$. She also did not have signs or symptoms of cerebral ischemia.

When the elder sibling reached $16 \mathrm{y}$ of age, both patients had been classified with familial steroid-resistant nephrotic syndrome of unknown genetic origin. Mutations in NPHS1, NPHS2 and Wilms tumor suppressor gene 1 had been previously excluded in both sisters. The diagnosis of SIOD was suggested by anthropometry and proven by genetic testing in patient 1 at the age of $16 \mathrm{y}$ and in patient 2 at the age of $11 \mathrm{y}$. Both patients lacked the following symptoms and signs of SIOD: facial and hair abnormalities, thorax deformation, waddling gait, naevi, cerebral symptoms, and vertebral deformation.

Anthropometric findings. At the age of $16 \mathrm{y}$, patient 1 presented with severe growth retardation (HT-SDS -4.7) (Fig. 1a), and a disproportion pattern typical for SIOD as described before by our working group (5). Detailed anthropometric analysis revealed a more severe reduction in sitting height (SH-SDS -5.8) than length of the extremities: AL-SDS -2.2 and LL-SDS -2.9 (Fig. 1b). The difference between her SH and $\mathrm{AL}$ was 3.6 SDS, contrary to only 0.3 SDS in the age-matched girls with CKD. The younger sister (patient 2) also showed severe height impairment at the age of $11 \mathrm{y}$ (Fig. 2a), however, the "typical" morphologic pattern of SIOD was
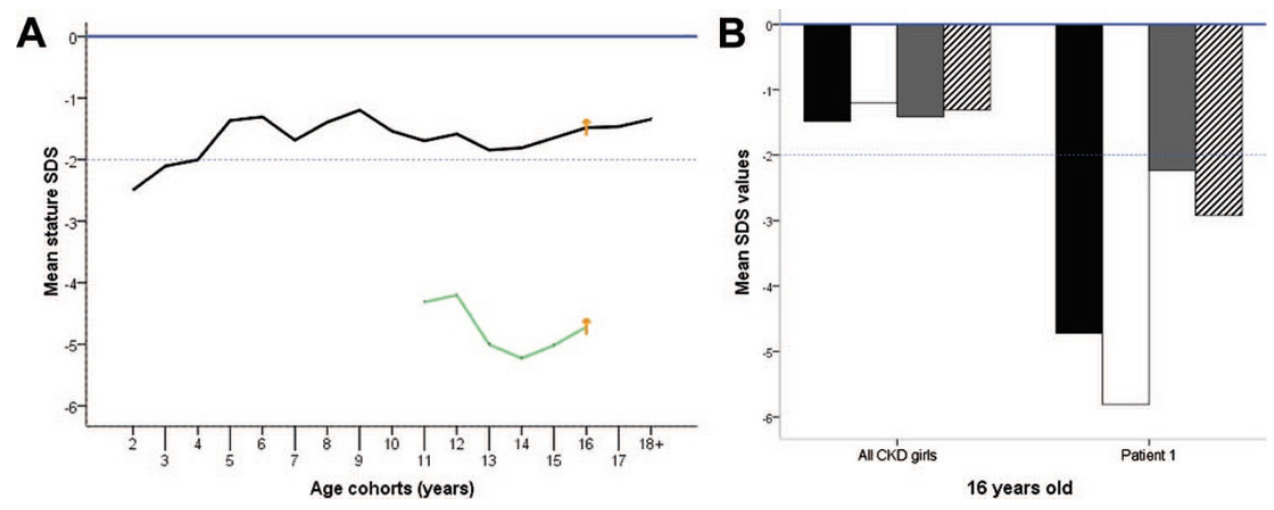

Figure 1. A) Height impairment by age in patient 1 with Schimke immuno-osseous dysplasia (SIOD) compared with 197 girls with chronic kidney disease stages (CKD) 2-5. black line, girls with CKD; green line, patient 1 with SIOD disease; arrows, age at which the longitudinal dimensions of patient 1 were compared with CKD patients. B) Pattern of the longitudinal dimensions in SIOD patient 1 compared with CKD patients at the age of 16 y. black bars, stature; open bars, sitting height; gray bars, arm length; hatched bars, leg length. 

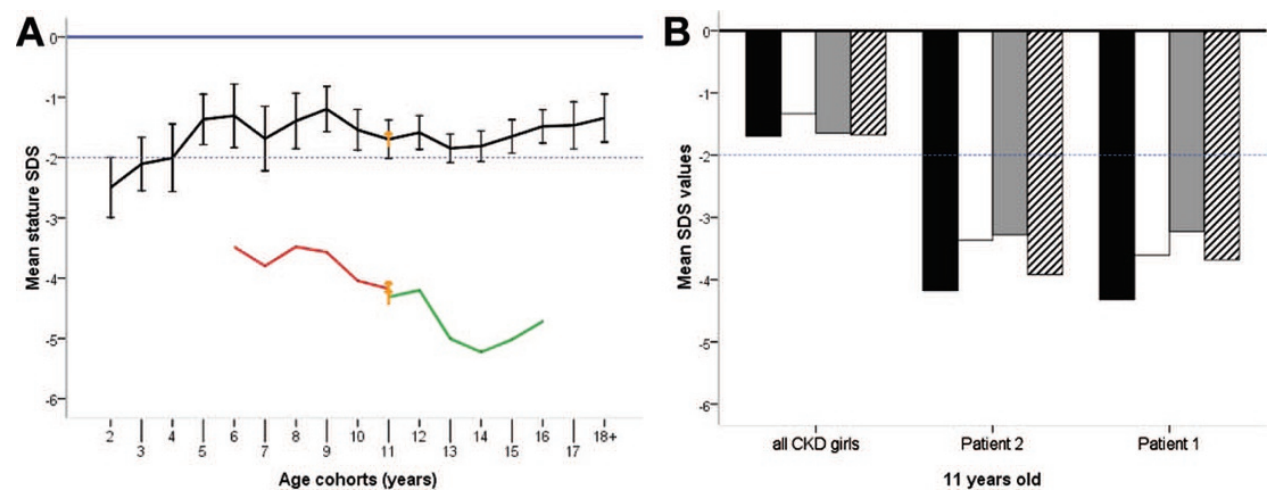

Figure 2. A) Height impairment by age in patient 2 with Schimke immuno-osseous dysplasia (SIOD) compared with 197 agematched girls with CKD stages $2-5$, and to patient 1. black line, girls with CKD; red line, patient 2 with SIOD; green line, patient 1 with SIOD; arrows, age at which the longitudinal dimensions of patient 1 were compared with CKD patients. B) Pattern of the longitudinal dimensions at the age of $11 \mathrm{y}, \mathrm{CKD}$ patients compared with SIOD patients 1 and 2. black bars, stature; open bars, sitting height; gray bars, arm length; hatched bars, leg length.

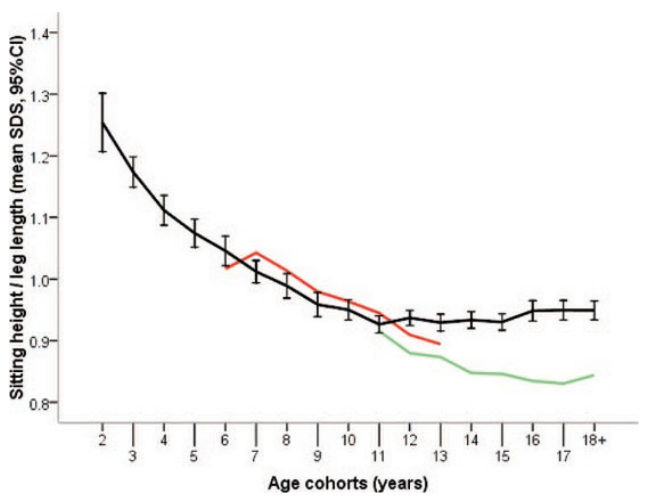

Figure 3. Sitting height/leg length ratio by age cohorts in patients 1 and 2 with SIOD vs 197 nonsyndromic girls with chronic kidney disease stages 2-5. black line, girls with CKD; green line, patient 1 with Schimke disease; red line, patient 2 with Schimke disease.

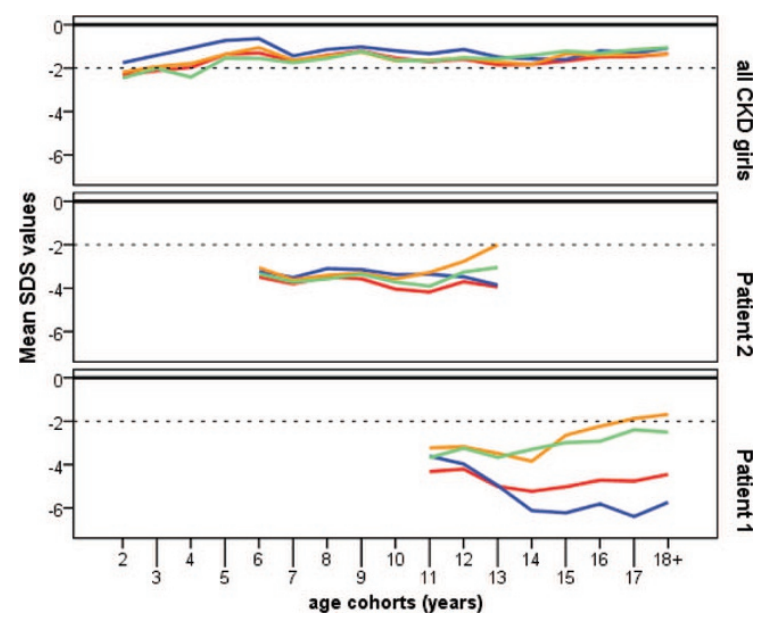

Figure 4. Longitudinal body dimensions by age cohorts in two girls with Schimke's disease (patient 1 and 2) vs 197 girls with chronic kidney disease stages 2-5. blue line, sitting height; red line, stature; green line, leg length; orange line, arm length.

not yet visible (Fig. 2b). Height, sitting height and length of extremities were almost identical in both girls at $11 \mathrm{y}$ of age. The sitting height/leg length ratio dramatically changed in both patients around the age of $12 \mathrm{y}$ and finally dropped out of the $95 \%$ confidence interval of mean for CKD females (Fig. 3).

As opposed to 197 girls with CKD of nonSIOD background, the disproportion of the two SIOD patients increased with age (Fig. 4).

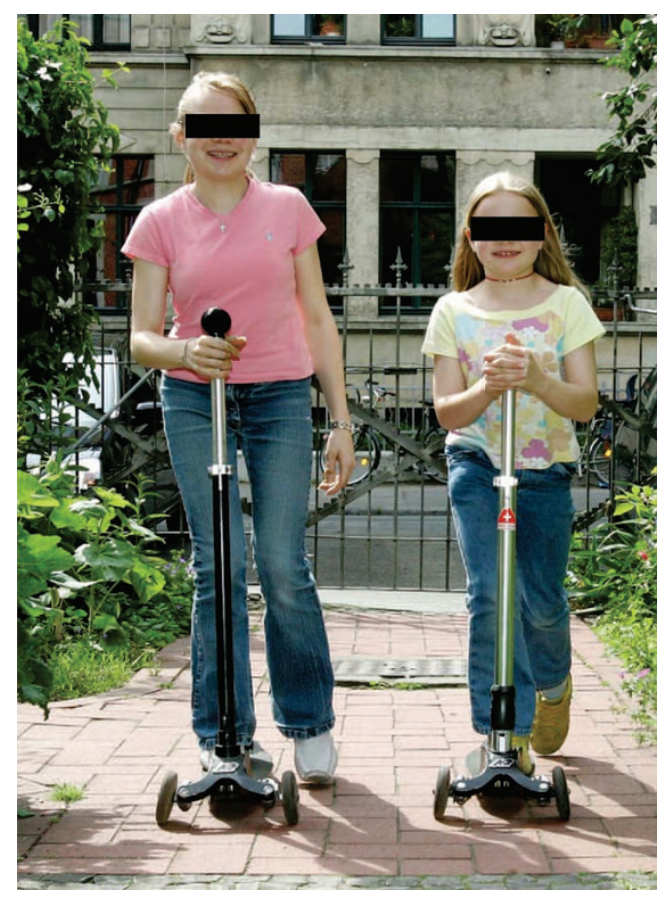

Figure 5. Photo of the two sisters with SIOD at 16 and $11 \mathrm{y}$ of age.

Figure 5 shows a photo of the two sisters at 11 and $16 \mathrm{y}$ of age.

Molecular genetics. Sequencing of the SMARCAL1 gene in both siblings revealed compound heterozygosity for two sequence alterations: a nonsense mutation in exon 17 (c.2542G $>$ T; p.E848 $\times$ ) and a missense change in exon 12 (c.1934G >A; p.R645H). The former is a known mutation that has been found recurrently in patients from various ethnic backgrounds (Finland, USA, Germany, Sweden, Czech Republic) (11), while the missense mutation in exon 12 is novel.

\section{DISCUSSION}

The main clinical findings leading to the diagnosis of SIOD are a combination of disproportionate growth failure, impaired cellular immune function, and steroid-resistant nephrotic syndrome (SRNS) with progressive renal failure $(1,4)$. Recently, we reported that SIOD patients show a characteristic anthropometric pattern distinguishing SIOD from nonSIOD chronic renal disease (5). The CKD patients without SIOD had a more 
significantly reduced leg length than trunk length while in SIOD patients the reduction in trunk length was significantly more pronounced than in leg length. However, our present data shows that mild phenotypes of SIOD, e.g., absence of thorax deformation, facial and skin abnormalities, etc. are difficult to diagnose and may even be overlooked because disproportion may be absent before the age of ten years.

As we did not follow-up our SIOD patients from birth we can only speculate on their pattern of growth before the age of six years. Our data starting at $6 y$ of age in patient 2 shows that this girl with a mild SIOD phenotype had a better sustained synchronicity of segmental growth than nonSIOD patients with CKD during prepubertal age. Her disproportion increased dramatically in adolescence, which is contrary to the growth pattern in females (Fig. 3 and 4) and males (12) with nonSIOD associated CKD.

In the siblings reported here, a mild phenotypic expression of SIOD was found to be associated with a new genotype consisting of compound-heterozygosity for a known nonsense mutation and a novel missense change. The recurrent mutation c. $2542 \mathrm{G}>\mathrm{T}$ (p.E848 $\times$ ) was previously shown to have arisen independently in different populations, suggesting that c.2542G is a hotspot for SIOD-associated SMARCAL1 mutations (11). The mutation probably leads to complete loss of function due to a truncated protein. In contrast, the mutant protein carrying the missense change p.R645H may retain some residual function. The highly conserved arginine at position 645 is located in a region of unknown function between the DEXH box and the helicase domain of the SMARCAL1 protein. A different change at the same position, p.R645C, was reported previously as a mutation causative for SIOD (4). The consistent clinical features together with the observation of disease-associated missense changes of neighboring amino acid residues (p.R644W, p.K647Q, p.K647T) $(4,11)$ are the best supportive evidence that p.R645H is indeed a causative mutation. Possibly, the substitution of the basic arginine by another basic residue (histidine) may have less severe consequences on the protein function compared with replacement by the neutral amino acid cysteine. It has been shown before that, compared with patients with biallelic nonsense, frameshift, or splicing mutations, SIOD patients with a missense mutation often have a milder disease regardless of whether the second allele encodes a nonsense or a missense mutation (11).

Our case report raises a number of other questions, one of which relates to the biopsy finding of minimal glomerular lesions (MCNS) instead of focal segmental glomerulosclerosis (FSGS), which is usually found in SIOD (1). However, Rottenberg et al. described MCNS in SIOD (13). FSGS could have been missed on a single biopsy in our patient if too few and no juxtamedullary glomeruli were sampled (14).

Another question deals with the fact that the younger sister did not undergo a renal biopsy and that her SRNS was not treated with an intensified immunosuppressive regimen. It was shown that children with familial SRNS with podocin mutations poorly responded to immunosuppressive therapies (15).
Furthermore, the findings of Boerkoel et al. (4) showed a lack of complete response to different immunosuppressive agents in children with SIOD. Therefore, the question arises whether all patients with SRNS should be evaluated by molecular genetic testing for gene mutations of SMARCAL1 before further immunosuppressive treatment can be started (14). This strategy will create considerable extra costs and may delay therapy in patients with idiopathic SRNS. Two predictive factors for a negative response to immunosuppressive therapy can easily be identified: 1) a history of congenital, infantile or familial SRNS indicates a high risk of a genetic type of SRNS; 2) growth failure preceding steroid therapy and exceeding the extent usually seen in CKD patients indicates a syndromic type of SRNS (5). If there are further findings showing dysproportionate growth failure, this will support the diagnosis of syndromic SRNS such as SIOD, nail-patella syndrome, and acroosteolysis.

We conclude from our observation that molecular genetic testing for SMARCAL1 should be considered for patients with steroid-resistant nephrotic syndrome and growth failure before steroid therapy and before advanced chronic kidney disease with other comorbidities including metabolic acidosis, renal osteodystrophy and malnutrition. This practice may be of special clinical importance to avoid overtreatment with immunosuppressive therapy in SIOD patients, who may suffer from life-threatening infections.

In addition, serial anthropometric assessment proved to be a valuable tool in distinguishing the SIOD growth pattern from CKD associated growth disturbance.

Our observation describes a mild form of SIOD and suggests that the mutation p.R645H is a hypomorphic allele.

Acknowledgments. We thank the patients and their parents for their cooperation and C.F. Boerkoel for his support and critical review of this manuscript.

\section{REFERENCES}

1. Ehrich JH, Offner G, Schirg E, Hoyer PF, Helmchen U, Brodehl J 1990 Association of spondylo-epiphyseal dysplasia with nephrotic syndrome. Pediatr Nephrol 4:117-121

2. Spranger J, Hinkel GK, Stoss H, Thoenes W, Wargowski D, Zepp F 1991 Schimke immuno-osseous dysplasia: a newly recognized multisystem disease. J Pediatr 119:64-72

3. Ehrich JH, Burchert W, Schirg E, Krull F, Offner G, Hoyer PF, Brodehl J 1995 Steroid- resistant nephrotic syndrome associated with spondyloepiphyseal dysplasia, transient ischemic attacks and lymphopenia. Clin Nephrol 43:89-95

4. Boerkoel CF, O’Neill S, Andre JL, Benke PJ, Bogdanovic R, Bulla M, Burguet A, Cockfield S, Cordeiro I, Ehrich JH, Frund S, Geary DF, Ieshima A, Illies F, Joseph MW, Kaitila I, Lama G, Leheup B, Ludman MD, McLeod DR, Medeira A, Milford DV, Ormala T, Rener-Primec Z, Weksberg R 2000 Manifestations and treatment of Schimke immuno-osseous dysplasia: 14 new cases and a review of the literature. Eur J Pediatr 159:1-7

5. Lücke T, Franke D, Clewing JM, Boerkoel CF, Ehrich JH, Das AM, Živičnjak M 2006 Schimke Versus Non-Schimke Chronic Kidney Disease: An Anthropometric Approach. Pediatrics 118:e400-e407

6. Gilchrist DM, Harley FL 1992 Schimke immuno-osseous dysplasia. J Pediatr 120:497

7. Hashimoto K, Takeuchi A, Ieshima A, Takada M, Kasagi M 1994 Juvenile variant of Schimke immunoosseous dysplasia. Am J Med Genet 49:266-269

8. Hogg RJ, Furth S, Lemley KV, Portman R, Schwartz GJ, Coresh J, Balk E, Lau J, Levin A, Kausz AT, Eknoyan G, Levey AS 2003 National Kidney Foundation's Kidney Disease Outcomes Quality Initiative clinical practice guidelines for chronic kidney disease in children and adolescents: evaluation, classification, and stratification. Pediatrics 111:1416-1421

9. Weiner JS, Lourie JA 1981 Practical Human Biology. London, United Kingdom: Academic Press 
10. Živičnjak M, Narančić NS, Szirovicza L, Franke D, Hrenović J, Bisof V 2003 Gender-specific growth patterns for stature, sitting height and limbs length in Croatian children and youth (3 to 18 years of age). Coll Antropol 27:321-334

11. Clewing JM, Fryssira H, Goodman D, Smithson SF, Sloan EA, Lou S, Huang Y, Choi K, Lücke T, Alpay H, André JL, Asakura Y, Biebuyck-Gouge N, Bogdanovic R, Bonneau D, Cancrini C, Cochat P, Cockfield S, Collard L, Cordeiro I, Cormier-Daire V, Cransberg K, Cutka K, Deschenes G, Ehrich JH, Fründ S, Georgaki H, Guillen-Navarro E, Hinkelmann B, Kanariou M, Kasap B, Kilic SS, Lama G, Lamfers P, Loirat C, Majore S, Milford D, Morin D, Ozdemir N, Pontz BF, Proesmans W, Psoni S, Reichenbach H, Reif S, Rusu C, Saraiva JM, Sakallioglu O, Schmidt B, Shoemaker L, Sigaudy S, Smith G, Sotsiou F, Stajic N, Stein A, Stray-Pedersen A, Taha D, Taque S, Tizard J, Tsimaratos M, Wong NA, Boerkoel CF 2007 Schimke immunoosseous dysplasia: suggestions of genetic diversity. Hum Mutat 28:273-283
12. Živičnjak M, Franke D, Filler G, Haffner D, Froede K, Nissel R, Haase S, Offner G, Ehrich JH, Querfeld U 2007 Growth impairment shows an age-dependent pattern in boys with chronic kidney disease. Pediatr Nephrol 22:420-429

13. Rottenberg GT, Shaw DG, Hall CM 1997 Spondyloepiphyseal dysplasia with nephrotic syndrome (Schimke immunoosseous dysplasia). J Pediatr Orthop B 6:7-10

14. Ehrich JH, Pape L, Schiffer M 2008 Corticosteroid-resistant nephrotic syndrome with focal and segmental glomerulosclerosis: an update of treatment options for children. Paediatr Drugs 10:9-22

15. Ruf RG, Lichtenberger A, Karle SM, Haas JP, Anacleto FE, Schultheiss M, Zalewski I, Imm A, Ruf EM, Mucha B, Bagga A, Neuhaus T, Fuchshuber A Hildebrandt F; Arbeitsgemeinschaft Fur Padiatrische Nephrologie Study Group 2004 Patients with mutations in NPHS2 (podocin) do not respond to standard steroid treatment of nephrotic syndrome. J Am Soc Nephrol 15:722-732 